\title{
Magnetic properties in ultrathin $3 d$ transition-metal binary alloys. II. Experimental verification of quantitative theories of damping and spin pumping
}

\author{
Martin A. W. Schoen, ${ }^{1,2}$ Juriaan Lucassen, ${ }^{3}$ Hans T. Nembach, ${ }^{1}$ Bert Koopmans, ${ }^{3}$ T. J. Silva, ${ }^{1}$ \\ Christian H. Back, ${ }^{2}$ and Justin M. Shaw ${ }^{1}$ \\ ${ }^{1}$ Quantum Electromagnetics Division, National Institute of Standards and Technology, Boulder, Colorado 80305, USA \\ ${ }^{2}$ Institute of Experimental and Applied Physics, University of Regensburg, 93053 Regensburg, Germany \\ ${ }^{3}$ Department of Applied Physics, Eindhoven University of Technology, PO Box 513, 5600 MB Eindhoven, The Netherlands
}

(Received 31 January 2017; published 7 April 2017)

\begin{abstract}
A systematic experimental study of Gilbert damping is performed via ferromagnetic resonance for the disordered crystalline binary $3 d$ transition-metal alloys $\mathrm{Ni}-\mathrm{Co}, \mathrm{Ni}-\mathrm{Fe}$, and $\mathrm{Co}-\mathrm{Fe}$ over the full range of alloy compositions. After accounting for inhomogeneous linewidth broadening, the damping shows clear evidence of both interfacial damping enhancement (by spin pumping) and radiative damping. We quantify these two extrinsic contributions and thereby determine the intrinsic damping. The comparison of the intrinsic damping to multiple theoretical calculations yields good qualitative and quantitative agreement in most cases. Furthermore, the values of the damping obtained in this study are in good agreement with a wide range of published experimental and theoretical values. Additionally, we find a compositional dependence of the spin mixing conductance.
\end{abstract}

DOI: 10.1103/PhysRevB.95.134411

\section{INTRODUCTION}

The magnetization dynamics in ferromagnetic films are phenomenologically well described by the Landau-LifshitzGilbert formalism (LLG) where the damping is described by a phenomenological damping parameter $\alpha$ [1,2]. Over the past four decades, there have been considerable efforts to derive the phenomenological damping parameter from first-principles calculations and to do so in a quantitative manner. One of the early promising theories was that of Kamberský, who introduced the so-called breathing Fermi surface model [3-5]. The name "breathing Fermi surface" stems from the picture that the precessing magnetization, due to spin-orbit coupling, distorts the Fermi surface. Repopulating the Fermi surface is delayed by the scattering time, resulting in a phase lag between the precession and the Fermi surface distortion. This lag leads to a damping that is proportional to the scattering time. Although this approach describes the so-called conductivitylike behavior of the damping at low temperatures, it fails to describe the high temperature behavior of some materials. The high temperature or resistivitylike behavior is described by the so-called "bubbling Fermi surface" model. In the case of energetically shifted bands, thermal broadening can lead to a significant overlap of the spin-split bands in $3 d$ ferromagnets. A precessing magnetization can induce electronic transitions between such overlapping bands, leading to spin-flip processes. This process scales with the amount of band overlap. Since such overlap is further increased with the band broadening that result from the finite temperature of the sample, this contribution is expected to increase as the temperature is increased. This model for interband transition mediated damping describes the resistivitylike behavior of the damping at higher temperatures (shorter scattering times). These two damping processes are combined in a torque correlation model by Gilmore et al. [6], as well as Thonig et al. [7], which describes both the low-temperature (intraband transitions) and high-temperature (interband transitions) behavior of the damping. Another approach via scattering theory was successfully implemented by Brataas et al. [8] to describe damping in transition metals. Starikov et al. [9] applied the scattering matrix approach to calculate the damping of $\mathrm{Ni}_{x} \mathrm{Fe}_{1-x}$ alloys and Liu et al. [10] expanded the formalism to include the influence of electron-phonon interactions.

A numerical realization of the torque correlation model was performed by Mankovsky et al. for $\mathrm{Ni}_{x} \mathrm{Co}_{1-x}, \mathrm{Ni}_{x} \mathrm{Fe}_{1-x}$, $\mathrm{Co}_{x} \mathrm{Fe}_{1-x}$, and $\mathrm{Fe}_{x} \mathrm{~V}_{1-x}$ [11]. More recently, Turek et al. [12] calculated the damping for $\mathrm{Ni}_{x} \mathrm{Fe}_{1-x}$ and $\mathrm{Co}_{x} \mathrm{Fe}_{1-x}$ alloys with the torque-correlation model, utilizing nonlocal torque correlators. It is important to stress that all of these approaches consider only the intrinsic damping. This complicates the quantitative comparison of calculated values for the damping to experimental data since there are many extrinsic contributions to the damping that result from sample structure, measurement geometry, and/or sample properties. While some extrinsic contributions to the damping and linewidth were discovered in the 1960s and 1970s, and are well described by theory, e.g., eddy-current damping [13,14], two-magnon scattering [15-17], the slow relaxer mechanism $[18,19]$, or radiative damping [20,21], interest in these mechanisms has been re-ignited recently $[22,23]$. Further contributions, such as spin pumping, both extrinsic [24,25] and intrinsic [24,26], have been discovered more recently and are subject to extensive research [27-31] for spintronics application. Therefore, in order to allow a quantitative comparison to theoretical calculations for intrinsic damping, both the measurement and sample geometry must be designed to allow both the determination and possible minimization of all additional contributions to the measured damping.

In this study we demonstrate methods to determine significant extrinsic contributions to the damping, which includes a measurement of the effective spin mixing conductance for both the pure elements and select alloys. By precisely accounting for all of these extrinsic contributions, we determine the intrinsic damping parameters of the binary alloys $\mathrm{Ni}_{x} \mathrm{Co}_{1-x}$, $\mathrm{Ni}_{x} \mathrm{Fe}_{1-x}$, and $\mathrm{Co}_{x} \mathrm{Fe}_{1-x}$ and compare them to the calculations by Mankovsky et al. [11], Turek et al., and Starikov et al. [9]. Furthermore, we present the concentration dependence 

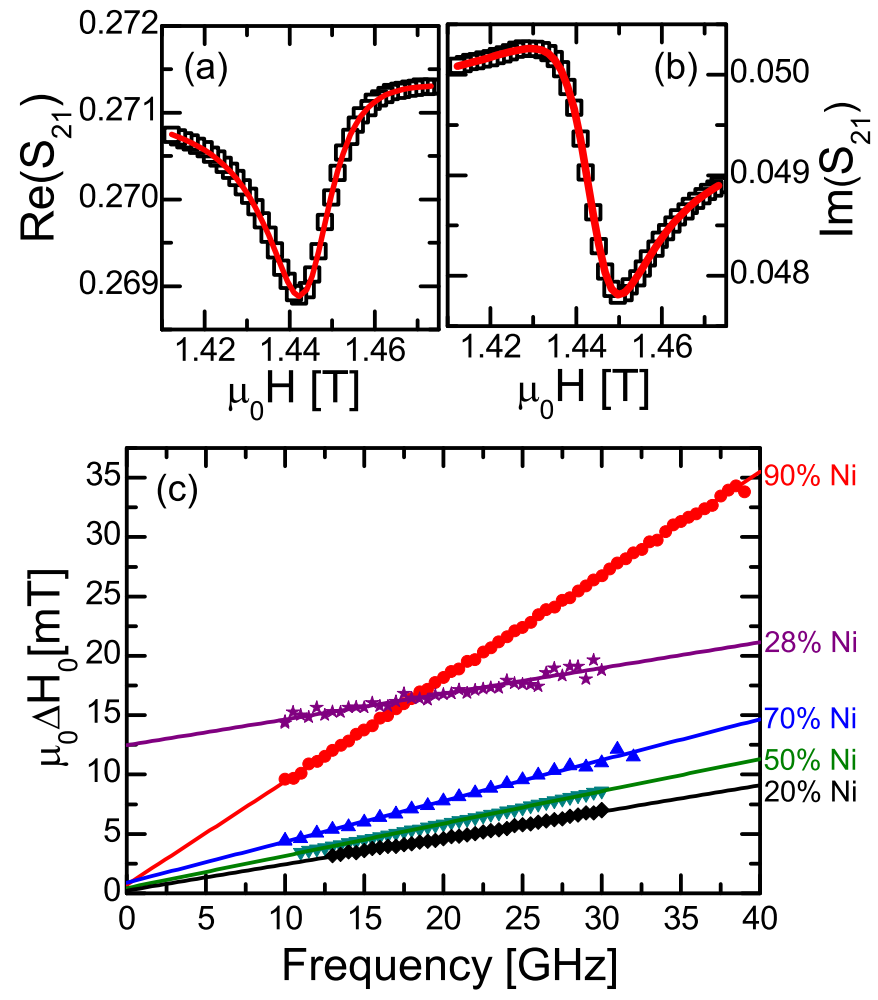

FIG. 1. (a) and (b) The real and imaginary part of the $S_{21}$ transmission parameter (black squares) measured at $20 \mathrm{GHz}$ with the complex susceptibility fit (red lines) for the $\mathrm{Ni}_{90} \mathrm{Fe}_{10}$ sample. (c) The linewidths from the susceptibility fits (symbols) and linear fits (solid lines) are plotted against frequency for different Ni-Fe compositions. Concentrations are denoted on the right-hand axis. The damping $\alpha$ and the inhomogeneous linewidth broadening $\Delta H_{0}$ for each alloy can be extracted from the fits via Eq. (1).

of the inhomogeneous linewidth broadening, which for most alloys shows exceptionally small values, indicative of the high homogeneity of our samples.

\section{SAMPLES AND METHOD}

We deposited $\mathrm{Ni}_{x} \mathrm{Co}_{1-x}, \mathrm{Ni}_{x} \mathrm{Fe}_{1-x}$, and $\mathrm{Co}_{x} \mathrm{Fe}_{1-x}$ alloys of varying composition (all compositions given in atomic percent) with a thickness of $10 \mathrm{~nm}$ on an oxidized (001) Si substrate with a $\mathrm{Ta}(3 \mathrm{~nm}) / \mathrm{Cu}(3 \mathrm{~nm})$ seed layer and a $\mathrm{Cu}(3 \mathrm{~nm}) / \mathrm{Ta}(3 \mathrm{~nm})$ cap layer. In order to investigate interface effects, we also deposited multiple thickness series at 10, 7, 4, 3 , and $2 \mathrm{~nm}$ of both the pure elements and select alloys. Structural characterization was performed using $\mathrm{x}$-ray diffraction (XRD). Field swept vector-network-analyzer ferromagnetic resonance spectroscopy (VNA-FMR) was used in the outof-plane geometry to determine the total damping parameter $\alpha_{\text {tot }}$. Further details of the deposition conditions, XRD, FMR measurement, and fitting of the complex susceptibility to the measured $S_{21}$ parameter are reported in Ref. [32].

An example of susceptibility fits to the complex $S_{21}$ data are shown in Figs. 1(a) and 1(b). All fits were constrained to a $3 \times$ linewidth $\Delta H$ field window around the resonance field in order to minimize the influence of measurement drifts on the error in the susceptibility fits. The total damping parameter $\alpha_{\text {tot }}$
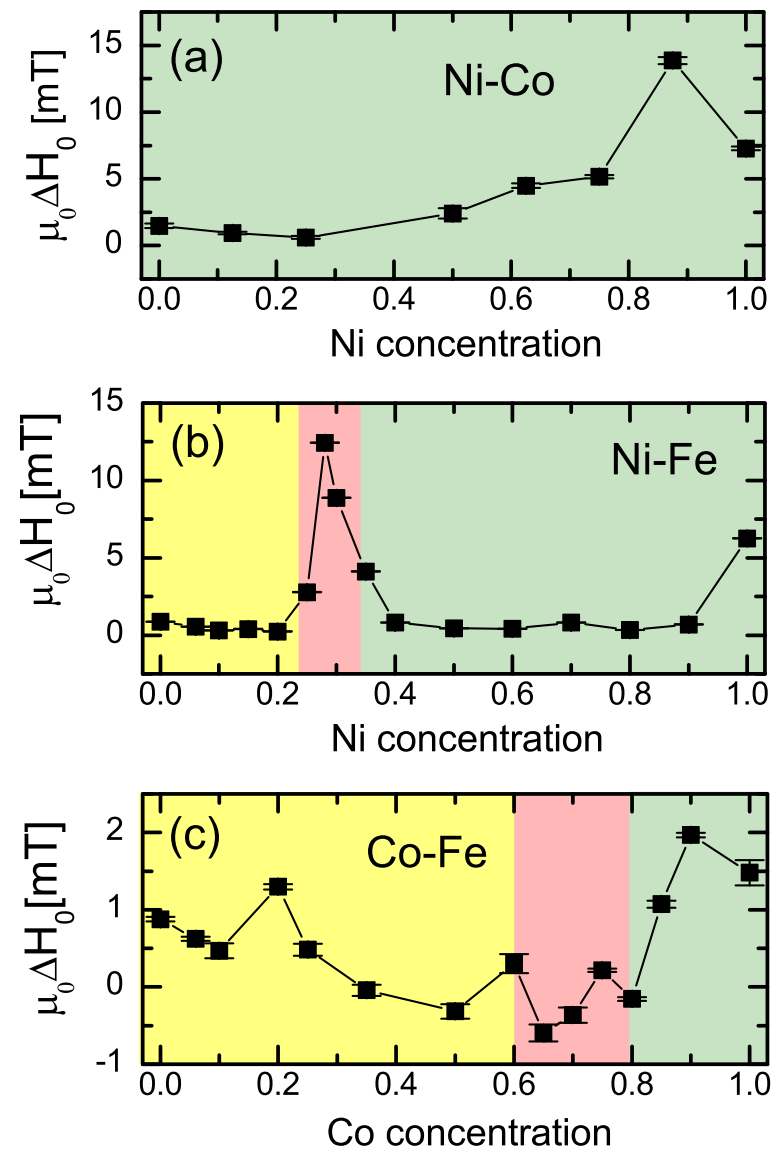

FIG. 2. The inhomogeneous linewidth-broadening $\Delta H_{0}$ is plotted vs alloy composition for (a) Ni-Co, (b) Ni-Fe, and (c) Co-Fe. The alloy phases are denoted by color code described in Ref. [32].

and the inhomogeneous linewidth broadening $\Delta H_{0}$ are then determined from a fit to the linewidth $\Delta H$ vs frequency $f$ plot [22], as shown in Fig. 1(c):

$$
\Delta H=\frac{4 \pi \alpha_{\mathrm{tot}} f}{\gamma \mu_{0}}+\Delta H_{0},
$$

where $\gamma=g \mu_{B} / \hbar$ is the gyromagnetic ratio, $\mu_{0}$ is the vacuum permeability, $\mu_{B}$ is the Bohr magneton, $\hbar$ is the reduced Planck constant, and $g$ is the spectroscopic $g$ factor reported in Ref. [32].

\section{RESULTS}

The first contribution to the linewidth we discuss is the inhomogeneous linewidth broadening $\Delta H_{0}$, which is presumably indicative of sample inhomogeneity [33,34]. We plot $\Delta H_{0}$ for all the alloy systems against the respective concentrations in Fig. 2. For all alloys, $\Delta H_{0}$ is in the range of a few $\mathrm{mT}$ to $10 \mathrm{mT}$. There are only a limited number of reports for $\Delta H_{0}$ in the literature with which to compare. For permalloy $\left(\mathrm{Ni}_{80} \mathrm{Fe}_{20}\right)$ we measure $\Delta H_{0}=0.35 \mathrm{mT}$, which is close to other reported values [35]. For the other $\mathrm{Ni}_{x} \mathrm{Fe}_{1-x}$ alloys, $\Delta H_{0}$ exhibits a significant peak near the fcc-to-bcc (face-centered-cubic to body-centered-cubic) phase transition at $30 \% \mathrm{Ni}$ [see Fig. 2(b)], which is easily seen in the raw data in Fig. 1(c). We speculate that this increase of inhomogeneous 
broadening in the $\mathrm{Ni}_{x} \mathrm{Fe}_{1-x}$ is caused by the coexistence of the bcc and fcc phases at the phase transition. However, the $\mathrm{Co}_{x} \mathrm{Fe}_{1-x}$ alloys do not exhibit an increase in $\Delta H_{0}$ at the equivalent phase transition at $70 \%$ Co. This suggests that the bcc and fcc phases of $\mathrm{Ni}_{x} \mathrm{Fe}_{1-x}$ tend to segregate near the phase transition, whereas the same phases for $\mathrm{Co}_{x} \mathrm{Fe}_{1-x}$ remain intermixed throughout the transition.

One possible explanation for inhomogeneous broadening is magnetic anisotropy, as originally proposed in Ref. [36]. However, this explanation does not account for our measured dependence of $\Delta H_{0}$ on alloy concentration, since the perpendicular magnetic anisotropy, described in Ref. [32], effectively exhibits opposite behavior with alloy concentration. An explanation for this is proposed in Ref. [37], where the additional effect of exchange narrowing is introduced to explain $\Delta H_{0}$ in thin Py films, where the exchange field is significantly larger than $\Delta H_{0}$. This assumption also holds for our alloys and indeed $\Delta H_{0}$ seems to roughly correlate to the inverse exchange constant [38,39].

We plot the total measured damping $\alpha_{\text {tot }}$ vs composition for $\mathrm{Ni}_{x} \mathrm{Co}_{1-x}, \mathrm{Ni}_{x} \mathrm{Fe}_{1-x}$, and $\mathrm{Co}_{x} \mathrm{Fe}_{1-x}$ in Fig. 3 (red crosses). The total damping of the $\mathrm{Ni}_{x} \mathrm{Co}_{1-x}$ system increases monotonically with increased $\mathrm{Ni}$ content. Such smooth behavior in the damping is not surprising owing to the absence of a phase transition for this alloy. In the $\mathrm{Ni}_{x} \mathrm{Fe}_{1-x}$ system, $\alpha_{\text {tot }}$ changes very little from pure $\mathrm{Fe}$ to approximately $25 \% \mathrm{Ni}$ where the bcc to fcc phase transition occurs. At the phase transition, $\alpha_{\text {tot }}$ exhibits a step, increasing sharply by approximately $30 \%$. For higher $\mathrm{Ni}$ concentrations, $\alpha_{\text {tot }}$ increases monotonically with increasing $\mathrm{Ni}$ concentration. On the other hand, the $\mathrm{Co}_{x} \mathrm{Fe}_{1-x}$ system shows a different behavior in the damping and displays a sharp minimum of $(2.3 \pm 0.1) \times 10^{-3}$ at $25 \%$ $\mathrm{Co}$ as previously reported [40]. As the system changes to an fcc phase $(\approx 70 \% \mathrm{Co}), \alpha_{\text {tot }}$ becomes almost constant.

We compare our data to previously published values in Table I. However, direct comparison of our data to previous reports is not trivial, owing to the variation in measurement conditions and sample characteristics for all the reported measurements. For example, the damping can depend on the temperature $[6,41]$. In addition, multiple intrinsic and intrinsic contributions to the total damping are not always accounted for in the literature. This can be seen in the fact that the reported damping in $\mathrm{Ni}_{80} \mathrm{Fe}_{20}$ (permalloy) varies from $\alpha=0.0055$ to $\alpha=0.04$ at room temperature among studies. The large variation for these reported data is possibly the result of different uncontrolled contributions to the extrinsic damping that add to the total damping in the different experiments, e.g., spin pumping [42-44] or roughness [43]. Therefore, the value for the intrinsic damping of $\mathrm{Ni}_{20} \mathrm{Fe}_{80}$ is expected to be at the low end of this scatter. Our measured value of $\alpha=0.0072$ lies within the range of reported values. Similarly, many of our measured damping values for different alloy compositions lie within the range of reported values [22,45-50]. Our measured damping of the pure elements and the $\mathrm{Ni}_{80} \mathrm{Fe}_{20}$ and $\mathrm{Co}_{90} \mathrm{Fe}_{10}$ alloys is compared to room temperature values found in literature in Table I, columns 2 and 3. Column 5 contains theoretically calculated values.

This scatter in the experimental data reported in the literature and its divergence from calculated values of the damping shows the necessity to determine the intrinsic damping $\alpha_{\text {int }}$ by quantification of all extrinsic contributions to the measured total damping $\alpha_{\text {tot }}$.

The first extrinsic contribution to the damping that we consider is the radiative damping $\alpha_{\text {rad }}$, which is caused by inductive coupling between sample and waveguide, which results in energy flow from the sample back into the microwave circuit [23]. $\alpha_{\text {rad }}$ depends directly on the measurement method and geometry. The effect is easily understood, since the strength of the inductive coupling depends on the inductance of the FMR mode itself, which is in turn determined by the saturation magnetization, sample thickness, sample length, and waveguide width. Assuming a homogeneous excitation field, a uniform magnetization profile throughout the sample, and negligible spacing between the waveguide and sample, $\alpha_{\text {rad }}$ is well approximated by [23]

$$
\alpha_{\mathrm{rad}}=\frac{\gamma M_{s} \mu_{0}^{2} t l}{16 Z_{0} w_{c c}},
$$

where $l(=10 \mathrm{~mm}$ in our case) is the sample length on the waveguide, $w_{\mathrm{cc}}(=100 \mu \mathrm{m})$ is the width of the coplanar waveguide center conductor, and $Z_{0}(=50 \Omega)$ the impedance of the waveguide. Though inherently small for most thin films, $\alpha_{\text {rad }}$ can become significant for alloys with exceptionally small intrinsic damping and/or high saturation magnetization. For example, it plays a significant role (values of $\alpha_{\text {rad }} \approx$ $5 \times 10^{-4}$ ) for the whole composition range of the Co-Fe alloy system and the Fe-rich side of the $\mathrm{Ni}-\mathrm{Fe}$ system. On the other hand, for pure $\mathrm{Ni}$ and permalloy $\left(\mathrm{Ni}_{80} \mathrm{Fe}_{20}\right) \alpha_{\text {rad }}$ comprises only $3 \%$ and $5 \%$ of $\alpha_{\text {tot }}$, respectively.

The second nonnegligible contribution to the damping that we consider is the interfacial contribution to the measured damping, such as spin pumping into the adjacent $\mathrm{Ta} / \mathrm{Cu}$ bilayers. Spin pumping is proportional to the reciprocal sample thickness as described in [24]

$$
\alpha_{\mathrm{sp}}=\frac{2 g_{\mathrm{eff}}^{\uparrow \downarrow} \mu_{B} g}{4 \pi M_{\mathrm{s}} t} .
$$

The spectroscopic $g$ factor and the saturation magnetization $M_{s}$ of the alloys were reported in Ref. [32] and the factor of 2 accounts for the presence of two nominally identical interfaces of the alloys in the cap and seed layers. In Figs. 4(a)-4(c) we plot the damping dependence on reciprocal thickness $1 / t$ for select alloy concentrations, which allows us to determine the effective spin mixing conductance $g_{\text {eff }}^{\uparrow \downarrow}$ through fits to Eq. (3). The effective spin mixing conductance contains details of the spin transport in the adjacent nonmagnetic layers, such as the interfacial spin mixing conductance, both the conductivity and spin diffusion for all the nonmagnetic layers with a nonnegligible spin accumulation, as well as the details of the spatial profile for the net spin accumulation [51,52]. The values of $g_{\text {eff }}^{\uparrow \downarrow}$ are plotted versus the alloy concentration in Fig. 4(d), and are in the range of previously reported values for samples prepared under similar growth conditions [51-55]. Intermediate values of $g_{\text {eff }}^{\uparrow \downarrow}$ are determined by a guide to the eye interpolation [gray lines, Fig. 4(d)] and $\alpha_{\text {sp }}$ is calculated for all alloy concentrations utilizing those interpolated values.

The data for $g_{\text {eff }}^{\uparrow \downarrow}$ in the $\mathrm{Ni}_{x} \mathrm{Fe}_{1-x}$ alloys show approximately a factor 2 increase of $g_{\text {eff }}^{\uparrow \downarrow}$ between Ni concentrations of $30 \%$ $\mathrm{Ni}$ and $50 \% \mathrm{Ni}$, which we speculate to occur at the fcc 

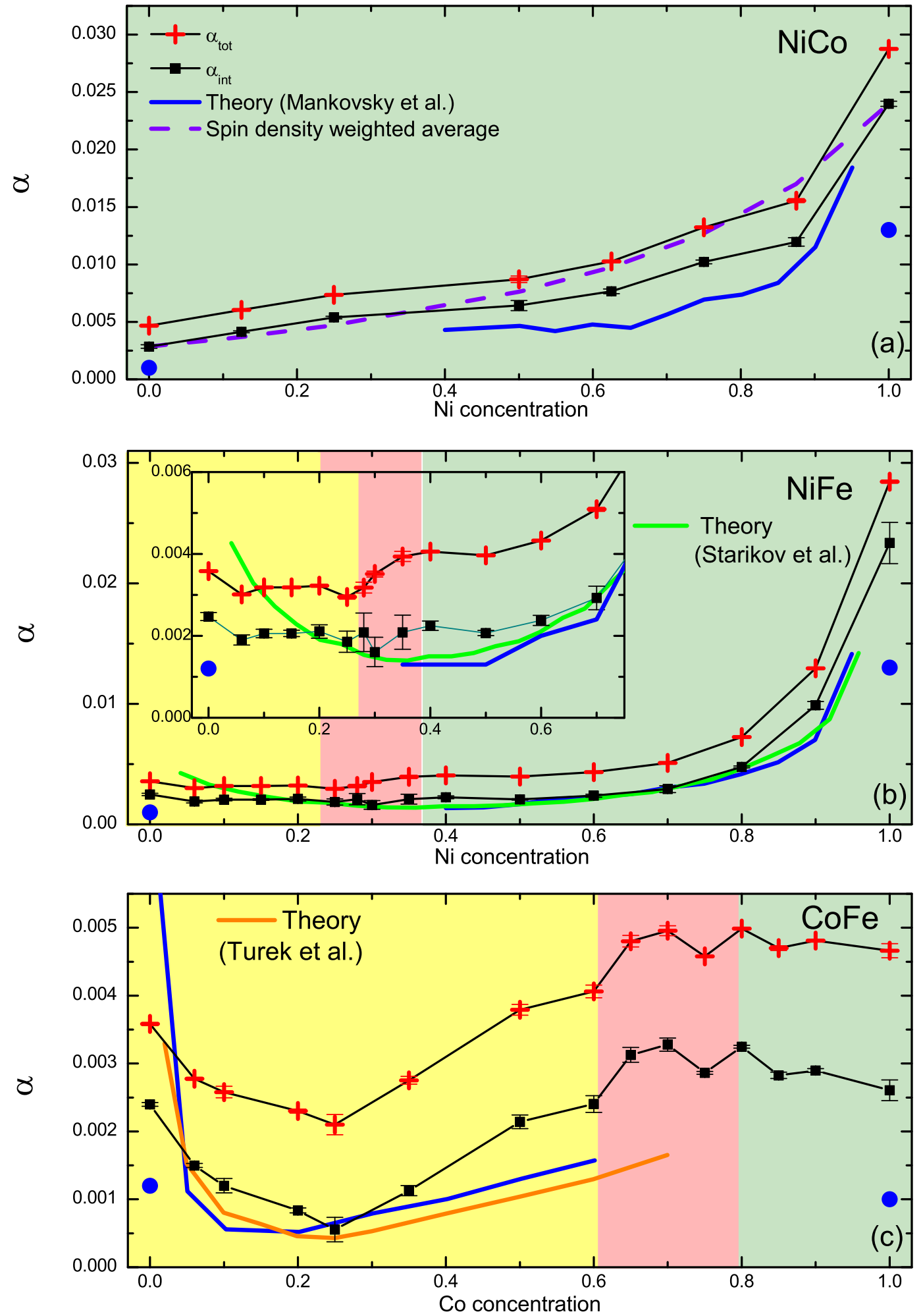

FIG. 3. The measured damping $\alpha_{\text {tot }}$ of all the alloys is plotted against the alloy composition (red crosses) for (a) Ni-Co, (b) Ni-Fe, and (c) $\mathrm{Co}-\mathrm{Fe}$ (the data in (c) are taken from Ref. [40]). The black squares are the intrinsic damping $\alpha_{\text {int }}$ after correction for spin pumping and radiative contributions to the measured damping. The blue line is the intrinsic damping calculated from the Ebert-Mankovsky theory [11], where the blue circles are the values for the pure elements at $300 \mathrm{~K}$. The green line is the calculated damping for the Ni-Fe alloys by Starikov et al. [9]. The inset in (b) depicts the damping in a smaller concentration window in order to better depict the small features in the damping around the phase transition. The damping for the Co-Fe alloys, calculated by Turek et al. [12], is plotted as the orange line. For the Ni-Co alloys the damping calculated by the spin density of the respective alloy weighted bulk damping [53] (purple dashed line). 
TABLE I. The total measured damping $\alpha_{\text {tot }}$ (col. 2) and the intrinsic damping (col. 4) for $\mathrm{Ni}_{80} \mathrm{Fe}_{20}, \mathrm{Co}_{90} \mathrm{Fe}_{10}$, and the pure elements are compared to both experimental (col. 3) and theoretical (col. 5) values from the literature. All values of the damping are at room temperature if not noted otherwise.

\begin{tabular}{|c|c|c|c|c|}
\hline Material & $\alpha_{\text {tot }}($ this study $)$ & Literature values & $\alpha_{\text {int }}$ (this study) & $\begin{array}{c}\text { Calculated literature } \\
\text { values }\end{array}$ \\
\hline $\mathrm{Ni}$ & 0.029 (fcc) & $\begin{array}{l}0.064[45] \\
0.045[56]\end{array}$ & 0.024 (fcc) & $\begin{array}{l}0.017[6](\mathrm{fcc}) \text { at } 0 \mathrm{~K} \\
0.022[10](\mathrm{fcc}) \text { at } 0 \mathrm{~K} \\
0.013[11](\mathrm{fcc})\end{array}$ \\
\hline $\mathrm{Fe}$ & 0.0036 (bcc) & $\begin{array}{l}0.0019[45] \\
0.0027[46]\end{array}$ & 0.0025 (bcc) & $\begin{array}{l}0.0013[6](\mathrm{bcc}) \text { at } 0 \mathrm{~K} \\
0.0010[10](\mathrm{bcc}) \text { at } 0 \mathrm{~K} \\
0.0012[11](\mathrm{bcc}) \text { at } 0 \mathrm{~K} \\
0.002[57](\mathrm{bcc})\end{array}$ \\
\hline $\mathrm{Co}$ & 0.0047 (fcc) & $0.011[45]$ & 0.0029 (fcc) & $\begin{array}{l}0.0011[6](\mathrm{hcp}) \text { at } 0 \mathrm{~K} \\
0.00073[10](\mathrm{hcp}) \text { at } 0 \mathrm{~K} \\
0.001[11](\mathrm{hcp})\end{array}$ \\
\hline $\mathrm{Ni}_{80} \mathrm{Fe}_{20}$ & $0.0073(\mathrm{fcc})$ & $\begin{array}{l}0.008[45] \\
0.008-0.04[58] \\
0.0078[47] \\
0.007[59] \\
0.006[60] \\
0.006[48] \\
0.0055[61]\end{array}$ & 0.0050 (fcc) & $\begin{array}{l}0.0046[9,62](\mathrm{fcc}) \text { at } 0 \mathrm{~K} \\
0.0039-0.0049[12](\mathrm{fcc}) \\
\text { at } 0 \mathrm{~K}\end{array}$ \\
\hline $\mathrm{Co}_{90} \mathrm{Fe}_{10}$ & 0.0048 (fcc) & $\begin{array}{l}0.0043[45] \\
0.0048[53]\end{array}$ & 0.0030 (fcc) & \\
\hline
\end{tabular}

to bec phase transition around $30 \% \mathrm{Ni}$. According to this line of speculation, the previously mentioned step increase in the measured total damping at the $\mathrm{Ni}_{x} \mathrm{Fe}_{1-x}$ phase transition can be fully attributed to the increase in spin pumping at the phase transition. In $\mathrm{Co}_{x} \mathrm{Fe}_{1-x}$, the presence of a step in $g_{\text {eff }}^{\uparrow \downarrow}$ at the phase transition is not confirmed, given the
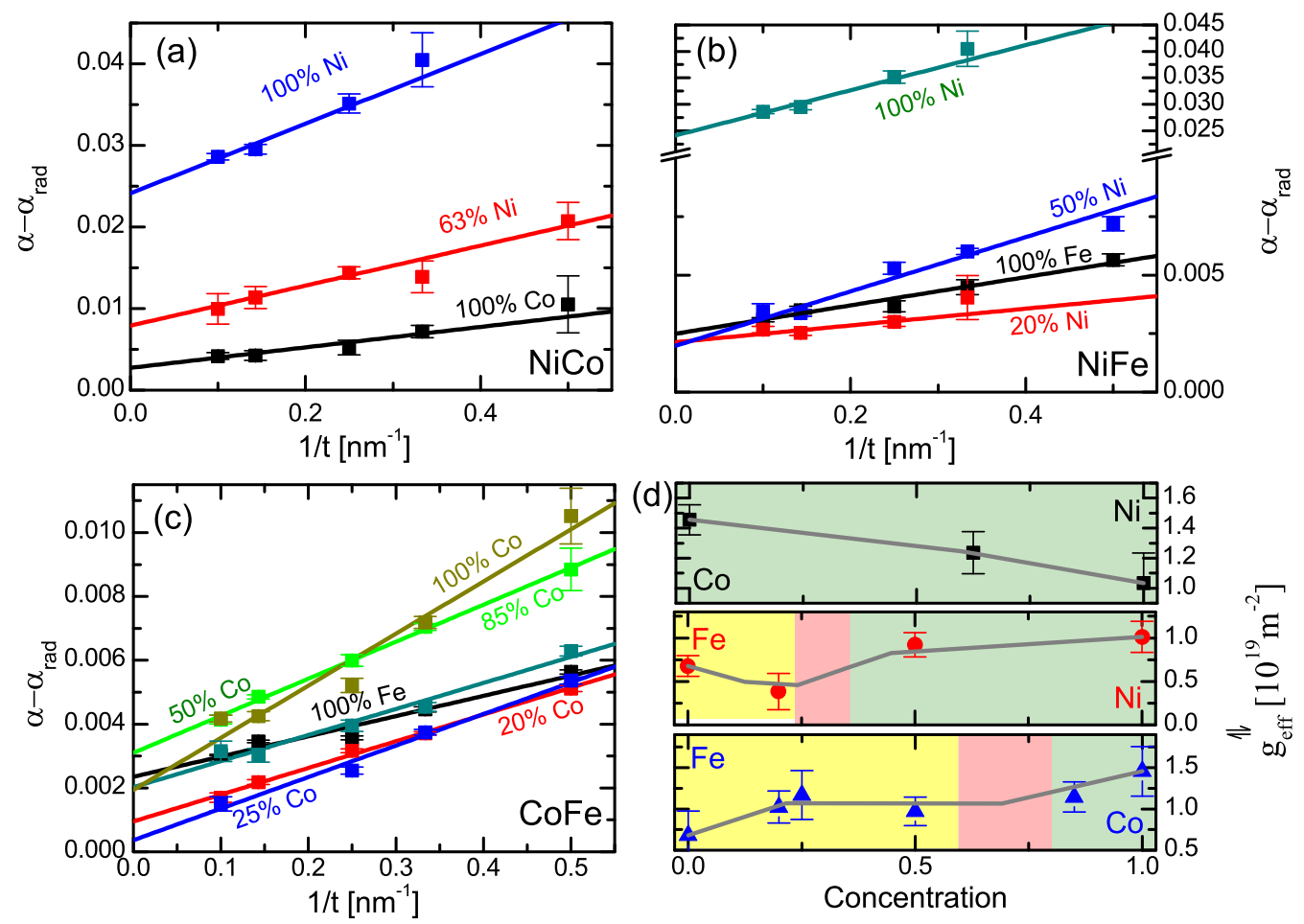

FIG. 4. The damping for the thickness series at select alloy compositions vs $1 / t$ for (a) Ni-Co, (b) Ni-Fe, and (c) Co-Fe (data points, concentrations denoted in the plots), with linear fits to Eq. (3) (solid lines). (d) The extracted effective spin mixing conductance $g_{\text {eff }}^{\uparrow \downarrow}$ for the measured alloy systems, where the gray lines show the linear interpolations for intermediate alloy concentrations. The data for the Co-Fe system are taken from Ref. [40]. 
measurement precision, although we do observe an increase in the effective spin mixing conductance when transitioning from the bcc to fcc phase. The concentration dependence of $g_{\text {eff }}^{\uparrow \downarrow}$ requires further thorough investigation and we therefore restrict ourselves to reporting the experimental findings.

Eddy-current damping $[13,14]$ is estimated by use of the equations given in Ref. [23] for films $10 \mathrm{~nm}$ thick or less. Eddy currents are neglected because they are found to be less than $5 \%$ of the total damping. Two-magnon scattering is disregarded because the mechanism is largely excluded in the out-of-plane measurement geometry [15-17]. The total measured damping is therefore well approximated as the sum

$$
\alpha_{\mathrm{tot}} \cong \alpha_{\mathrm{int}}+\alpha_{\mathrm{rad}}+\alpha_{\mathrm{sp}}
$$

We determine the intrinsic damping of the material by subtracting $\alpha_{\mathrm{sp}}$ and $\alpha_{\text {rad }}$ from the measured total damping, as shown in Fig. 3.

The intrinsic damping increases monotonically with $\mathrm{Ni}$ concentration for the $\mathrm{Ni}_{x} \mathrm{Co}_{1-x}$ alloys. Indicative of the importance of extrinsic sources of damping, $\alpha_{\text {int }}$ is approximately $40 \%$ smaller than $\alpha_{\text {tot }}$ for the Co-rich alloy, though the difference decreases to only $15 \%$ for pure Ni. This behavior is expected, given that $\alpha_{\text {rad }}$ and $\alpha_{\mathrm{sp}}$ are, respectively, proportional and inversely proportional to $M_{s}$. A comparison of $\alpha_{\text {int }}$ to the calculations by Mankovsky et al. [11] shows excellent quantitative agreement to within 30\%. Furthermore, we compare $\alpha_{\text {int }}$ of the $\mathrm{Ni}_{x} \mathrm{Co}_{1-x}$ alloys to the spin density weighted average of the intrinsic damping of $\mathrm{Ni}$ and $\mathrm{Co}$ [purple dashed line in Fig. 3(a)], which gives good agreement with our data, as previously reported [53].

$\alpha_{\text {int }}$ for $\mathrm{Ni}_{x} \mathrm{Fe}_{1-x}$ [Fig. 3(b)] also increases with $\mathrm{Ni}$ concentration after a small initial decrease from pure Fe to the first $\mathrm{Ni}_{x} \mathrm{Fe}_{1-x}$ alloys. The step increase found in $\alpha_{\text {tot }}$ at the bcc to fcc phase transition is fully attributed to $\alpha_{\mathrm{sp}}$, as detailed in the previous section, and therefore does not occur in $\alpha_{\text {int }}$. Similar to the $\mathrm{Ni}_{x} \mathrm{Co}_{1-x}$ system $\alpha_{\text {int }}$ is significantly lower than $\alpha_{\text {tot }}$ for Fe-rich alloys. Within error bars, a comparison to the calculations by Mankovsky et al. [11] (blue line) and Starikov et al. [9] (green line) exhibit excellent agreement in the fcc phase, with marginally larger deviations in the Ni-rich regime. Starikov et al. [9] calculated the damping over the full range of compositions, under the assumption of continuous fcc phase. This calculation deviates further from our measured $\alpha_{\text {int }}$ in the bcc phase exhibiting qualitatively different behavior.

As previously reported, the dependence of $\alpha_{\text {int }}$ on alloy composition in the $\mathrm{Co}_{x} \mathrm{Fe}_{1-x}$ alloys exhibits strongly nonmonotonic behavior, differing from the two previously discussed alloys [40]. $\alpha_{\text {int }}$ displays a minimum at $25 \%$ Co concentration with a, for conducting ferromagnets unprecedented, low value of $\alpha_{\text {int }}=(5 \pm 1.8) \times 10^{-4}$. With increasing Co concentration, $\alpha_{\text {int }}$ grows up to the phase transition, at which point it increases by $10 \%$ to $20 \%$ until it reaches the value for pure Co. It was shown that $\alpha_{\text {int }}$ scales with the density of states (DOS) at the Fermi energy $n\left(E_{\mathrm{F}}\right)$ in the bcc phase [40], and the DOS also exhibits a sharp minimum for $\mathrm{Co}_{25} \mathrm{Fe}_{75}$. This scaling is expected $[63,64]$ if the damping is dominated by the breathing Fermi surface process. With the breathing surface model, the intraband scattering that leads to damping directly scales with $n\left(E_{\mathrm{F}}\right)$. This scaling is particularly pronounced in the Co-Fe alloy system due to the small concentration dependence of the spin-orbit coupling on alloy composition. The special properties of the $\mathrm{Co}_{x} \mathrm{Fe}_{1-x}$ alloy system are discussed in greater detail in Ref. [40].

Comparing $\alpha_{\text {int }}$ to the calculations by Mankovsky et al. [11], we find good quantitative agreement with the value of the minimum. However, the concentration of the minimum is calculated to occur at approximately $10 \%$ to $20 \% \mathrm{Co}$, a slightly lower value than $25 \%$ Co that we find in this study. Furthermore, the strong concentration dependence around the minimum is not reflected in the calculations. More recent calculations by Turek et al. [12] for the bcc $\mathrm{Co}_{x} \mathrm{Fe}_{1-x}$ alloys [orange line in Fig. 3(c)] find the minimum of the damping of $4 \times 10^{-4}$ at $25 \%$ Co concentration in good agreement with our experiment, but there is some deviation in concentration dependence of the damping around the minimum. Turek et al. [12] also reported on the damping in the $\mathrm{Ni}_{x} \mathrm{Fe}_{1-x}$ alloy system, with similar qualitative and quantitative results as the other two presented quantitative theories [9,11] and the results are therefore not plotted in Fig. 3(b) for the sake of comprehensibility of the figure. For both $\mathrm{Ni}_{x} \mathrm{Fe}_{1-x}$ and the $\mathrm{Co}_{x} \mathrm{Fe}_{1-x}$ alloys, the calculated spin density weighted intrinsic damping of the pure elements (not plotted) deviates significantly from the determined intrinsic damping of the alloys, contrary to the good agreement archived for the $\mathrm{Co}_{x} \mathrm{Ni}_{1-x}$ alloys. We speculate that this difference between the alloy systems is caused by the nonmonotonous dependence of the density of states at the Fermi energy in the $\mathrm{Co}_{x} \mathrm{Fe}_{1-x}$ and $\mathrm{Ni}_{x} \mathrm{Fe}_{1-x}$ systems.

Other calculated damping values for the pure elements and the $\mathrm{Ni}_{80} \mathrm{Fe}_{20}$ and $\mathrm{CO}_{90} \mathrm{Fe}_{10}$ alloys are compared to the determined intrinsic damping in Table I. Generally, the calculations underestimate the damping significantly, but our data are in good agreement with more recent calculations for permalloy $\left(\mathrm{Ni}_{80} \mathrm{Fe}_{20}\right)$ [62].

It is important to point out that none of the theories considered here include thermal fluctuations. Regardless, we find exceptional agreement with the calculations to $\alpha_{\text {int }}$ at intermediate alloy concentrations. We speculate that the modeling of atomic disorder in the alloys in the calculations, by the coherent potential approximation (CPA), could be responsible for this exceptional agreement. The effect of disorder on the electronic band structure possibly dominates any effects due to nonzero temperature. Indeed, both effects cause a broadening of the bands due to enhanced momentum scattering rates. This directly correlates to a change of the damping parameter according to the theory of Gilmore and Stiles [6]. Therefore, the inclusion of the inherent disorder of solid-solution alloys in the calculations by Mankovsky et al. [11] mimics the effects of temperature on damping to some extent. This argument is corroborated by the fact that the calculations by Mankovsky et al. [11] diverge for diluted alloys and pure elements [as shown in Fig. 3(c) for pure $\mathrm{Fe}$, where no or to little disorder is introduced to account for temperature effects. Mankovsky et al. [11] performed temperature dependent calculations of the damping for pure bcc $\mathrm{Fe}$, fcc $\mathrm{Ni}$, and hcp $\mathrm{Co}$ and the values for $300 \mathrm{~K}$ are shown in Table I and Fig. 3. These calculations for $\alpha_{\text {int }}$ at a temperature of $300 \mathrm{~K}$ are approximately a factor of 2 less than our measured values, but the agreement is significantly 


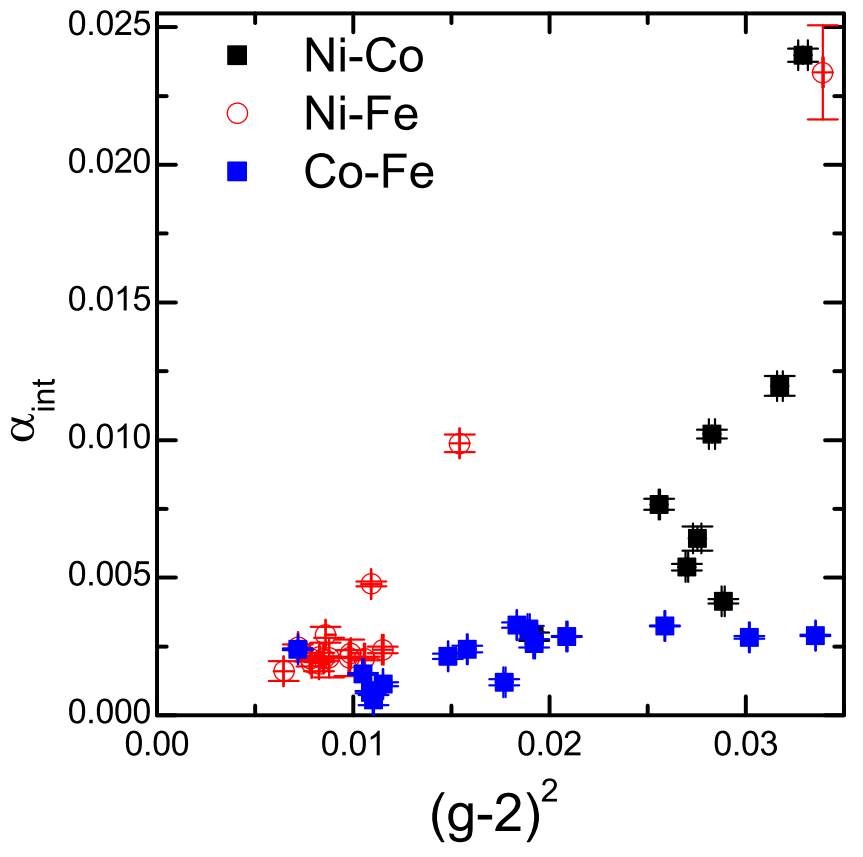

FIG. 5. The intrinsic damping $\alpha_{\text {int }}$ is plotted against $(g-2)^{2}$ for all alloys. We do not observe a proportionality between $\alpha_{\text {int }}$ and $(g-2)^{2}$.

improved relative to those obtained by calculations that neglect thermal fluctuations.

Finally, it has been reported $[50,65]$ that there is a general proportionality between $\alpha_{\text {int }}$ and $(g-2)^{2}$, as contained in the original microscopic BFS model proposed by Kamberský [66]. To examine this relationship, we plot $\alpha_{\text {int }}$ versus $(g-2)^{2}$ (determined in Ref. [32]) for all samples measured here in Fig. 5. While some samples with large values for $(g-2)^{2}$ also exhibit large $\alpha_{\text {int }}$, this is not a general trend for all the measured samples. Given that the damping is not purely a function of the spin-orbit strength, but also depends on the details of the band structure, the result in Fig. 5 is expected. For example, the amount of band overlap will determine the amount of interband transition leading to that damping channel. Furthermore, the density of states at the Fermi energy will affect the intraband contribution to the damping [6,7]. Finally, the ratio of interto intraband scattering that mediates damping contributions at a fixed temperature (RT for our measurements) changes for different elements [6,7] and therefore with alloy concentration. None of these factors are necessarily proportional to the spin-orbit coupling. Therefore, we conclude that this simple relation, which originally traces to an order of magnitude estimate for the case of spin relaxation in semiconductors [67], does not hold for all magnetic systems in general.

\section{SUMMARY}

We determined the damping for the full composition range of the binary $3 d$ transition-metal alloys Ni-Co, Ni-Fe, and Co$\mathrm{Fe}$ and showed that the measured damping can be explained by three contributions to the damping: intrinsic damping, radiative damping, and damping due to spin pumping. By quantifying all extrinsic contributions to the measured damping, we determine the intrinsic damping over the whole range of alloy compositions. These values are compared to multiple theoretical calculations and yield excellent qualitative and good quantitative agreement for intermediate alloy concentrations. For pure elements or diluted alloys, the effect of temperature seems to play a larger role for the damping and calculations including temperature effects give significantly better agreement to our data. Furthermore, we demonstrated a compositional dependence of the spin mixing conductance, which can vary by a factor of 2 . Finally, we showed that the often postulated dependence of the damping on the $g$ factor does not apply to the investigated binary alloy systems, as their damping cannot be described solely by the strength of the spin-orbit interaction.
[1] T. L. Gilbert, A phenomenological theory of damping in ferromagnetic materials, IEEE Trans. Mag. 40, 3443 (2004).

[2] L. D. Landau and E. M. Lifshitz, Theory of the dispersion of magnetic permeability in ferromagnetic bodies, Phys. Z. Sowietunion 8, 153 (1935).

[3] V. Kamberský, FMR linewidth and disorder in metals, Czech. J. Phys. B 34, 1111 (1984).

[4] V. Kamberský, On ferromagnetic resonance damping in metals, Czech. J. Phys. B 26, 1366 (1976).

[5] V. Kamberský and C. E. Patton, Spin-wave relaxation and phenomenological damping in ferromagnetic resonance, Phys. Rev. B 11, 2668 (1975).

[6] K. Gilmore, Y. U. Idzerda, and M. D. Stiles, Identification of the Dominant Precession-Damping Mechanism in Fe, Co, and Ni by First-Principles Calculations, Phys. Rev. Lett. 99, 027204 (2007).

[7] D. Thonig and J. Henk, Gilbert damping tensor within the breathing Fermi surface model: Anisotropy and non-locality, New J. Phys. 16, 013032 (2014).
[8] A. Brataas, Y. Tserkovnyak, and G. E. W. Bauer, Scattering Theory of Gilbert Damping, Phys. Rev. Lett. 101, 037207 (2008).

[9] A. A. Starikov, P. J. Kelly, A. Brataas, Y. Tserkovnyak, and G. E. W. Bauer, Unified First-Principles Study of Gilbert Damping, Spin-Flip Diffusion, and Resistivity in Transition Metal Alloys, Phys. Rev. Lett. 105, 236601 (2010).

[10] Y. Liu, A. A. Starikov, Z. Yuan, and P. J. Kelly, First-principles calculations of magnetization relaxation in pure $\mathrm{Fe}, \mathrm{Co}$, and $\mathrm{Ni}$ with frozen thermal lattice disorder, Phys. Rev. B 84, 014412 (2011).

[11] S. Mankovsky, D. Ködderitzsch, G. Woltersdorf, and H. Ebert, First-principles calculation of the Gilbert damping parameter via the linear response formalism with application to magnetic transition metals and alloys, Phys. Rev. B 87, 014430 (2013).

[12] I. Turek, J. Kudrnovský, and V. Drchal, Nonlocal torque operators in ab initio theory of the Gilbert damping in random ferromagnetic alloys, Phys. Rev. B 92, 214407 (2015). 
[13] J. M. Lock, Eddy current damping in thin metallic ferromagnetic films, Br. J. Appl. Phys. 17, 1645 (1966).

[14] P. Pincus, Excitation of spin waves in ferromagnets: Eddy current and boundary condition effects, Phys. Rev. 118, 658 (1960).

[15] M. J. Hurben and C. E. Patton, Theory of two magnon scattering microwave relaxation and ferromagnetic resonance linewidth in magnetic thin films, J. Appl. Phys. 83, 4344 (1998).

[16] J. Lindner, K. Lenz, E. Kosubek, K. Baberschke, D. Spoddig, R. Meckenstock, J. Pelzl, Z. Frait, and D. L. Mills, NonGilbert-type damping of the magnetic relaxation in ultrathin ferromagnets: Importance of magnon-magnon scattering, Phys. Rev. B 68, 060102 (2003).

[17] M. Sparks, R. Loudon, and C. Kittel, Ferromagnetic relaxation. I. Theory of the relaxation of the uniform precession and the degenerate spectrum in insulators at low temperatures, Phys. Rev. 122, 791 (1961).

[18] J. F. Dillon and J. W. Nielsen, Effects of Rare Earth Impurities on Ferrimagnetic Resonance in Yttrium Iron Garnet, Phys. Rev. Lett. 3, 30 (1959).

[19] J. H. Van Vleck and R. Orbach, Ferrimagnetic Resonance of Dilute Rare-Earth Doped Iron Garnets, Phys. Rev. Lett. 11, 65 (1963).

[20] R. W. Sanders, D. Paquette, V. Jaccarino, and S. M. Rezende, Radiation damping in magnetic resonance. II. Continuous-wave antiferromagnetic-resonance experiments, Phys. Rev. B 10, 132 (1974).

[21] G. Wende, Radiation damping in FMR measurements in a nonresonant rectangular waveguide, Phys. Status Solidi (a) 36, 557 (1976).

[22] H. T. Nembach, T. J. Silva, J. M. Shaw, M. L. Schneider, M. J. Carey, S. Maat, and J. R. Childress, Perpendicular ferromagnetic resonance measurements of damping and Lande $g$ factor in sputtered $\left(\mathrm{Co}_{2} \mathrm{Mn}_{1-x} \mathrm{Ge}_{x}\right.$ films, Phys. Rev. B 84, 054424 (2011).

[23] M. A. W. Schoen, J. M. Shaw, H. T. Nembach, M. Weiler, and T. J. Silva, Radiative damping in waveguide-based ferromagnetic resonance measured via analysis of perpendicular standing spin waves in sputtered permalloy films, Phys. Rev. B 92, 184417 (2015).

[24] Y. Tserkovnyak, A. Brataas, and G. E. W. Bauer, Enhanced Gilbert Damping in Thin Ferromagnetic Films, Phys. Rev. Lett. 88, 117601 (2002).

[25] Y. Tserkovnyak, A. Brataas, G. E. W. Bauer, and B. I. Halperin, Nonlocal magnetization dynamics in ferromagnetic heterostructures, Rev. Mod. Phys. 77, 1375 (2005).

[26] V. G. Bar'yakhtar, E. B. Krotenko, and D. A. Yablonskii, Magnetic symmetry of domain walls with Bloch lines in ferromagnets and ferrites, JETP 64, 542 (1986).

[27] M. Haertinger, C. H. Back, J. Lotze, M. Weiler, S. Geprags, H. Huebl, S. T. B. Goennenwein, and G. Woltersdorf, Spin pumping in YIG/Pt bilayers as a function of layer thickness, Phys. Rev. B 92, 054437 (2015).

[28] S. Lequeux et al., Increased magnetic damping of a single domain wall and adjacent magnetic domains detected by spin torque diode in a nanostripe, Appl. Phys. Lett. 107, 182404 (2015).

[29] Y. Li and W. E. Bailey, Wave-Number-Dependent Gilbert Damping in Metallic Ferromagnets, Phys. Rev. Lett. 116, 117602 (2016).
[30] H. T. Nembach, J. M. Shaw, C. T. Boone, and T. J. Silva, Modeand Size-Dependent Landau-Lifshitz Damping in Magnetic Nanostructures: Evidence for Nonlocal Damping, Phys. Rev. Lett. 110, 117201 (2013).

[31] T. Weindler, H. G. Bauer, R. Islinger, B. Boehm, J. Y. Chauleau, and C. H. Back, Magnetic Damping: Domain Wall Dynamics versus Local Ferromagnetic Resonance, Phys. Rev. Lett. 113, 237204 (2014).

[32] M. A. W. Schoen, J. Lucassen, H. T. Nembach, T. J. Silva, B. Koopmans, C. H. Back, and J. M. Shaw, Magnetic properties of ultrathin $3 \mathrm{~d}$ transition-metal binary alloys. I. Spin and orbital moments, anisotropy, and confirmation of Slater-Pauling behavior, Phys. Rev. B 95, 134410 (2017).

[33] Z. Celinski and B. Heinrich, Ferromagnetic resonance linewidth of Fe ultrathin films grown on a bcc $\mathrm{Cu}$ substrate, J. Appl. Phys. 70, 5935 (1991).

[34] W. Platow, A. N. Anisimov, G. L. Dunifer, M. Farle, and K. Baberschke, Correlations between ferromagnetic-resonance linewidths and sample quality in the study of metallic ultrathin films, Phys. Rev. B 58, 5611 (1998).

[35] D. J. Twisselmann and R. D. McMichael, Intrinsic damping and intentional ferromagnetic resonance broadening in thin Permalloy films, J. Appl. Phys. 93, 6903 (2003).

[36] R. D. McMichael, D. J. Twisselmann, and A. Kunz, Localized Ferromagnetic Resonance in Inhomogeneous Thin Films, Phys. Rev. Lett. 90, 227601 (2003).

[37] H. Hurdequint, FMR studies of ultrathin permalloy layers sandwiched by $\mathrm{Al}_{2} \mathrm{O}_{3}$, J. Magn. Magn. Mater. 242-245, 521 (2002).

[38] O. D. Hennemann and E. Siegel, Spin-wave measurements of exchange constant A in Ni-Fe alloy thin films, Phys. Status Solidi 77, 229 (1976).

[39] C. Wilts and S. Lai, Spin wave measurements of exchange constant in Ni-Fe alloy films, IEEE Trans. Magn. 8, 280 (1972).

[40] M. A. W. Schoen et al., Ultra-low magnetic damping of a metallic ferromagnet, Nat. Phys. 12, 839 (2016).

[41] B. Heinrich, D. J. Meredith, and J. F. Cochran, Wave number and temperature dependent Landau-Lifshitz damping in nickel, J. Appl. Phys. 50, 7726 (1979).

[42] L. Lagae, R. Wirix-Speetjens, W. Eyckmans, S. Borghs, and J. D. Boeck, Increased Gilbert damping in spin valves and magnetic tunnel junctions, J. Magn. Magn. Mater. 286, 291 (2005).

[43] J. O. Rantschler et al., Damping at normal metal/permalloy interfaces, Magn., IEEE Trans. 41, 3523 (2005).

[44] J. M. Shaw, H. T. Nembach, and T. J. Silva, Determination of spin pumping as a source of linewidth in sputtered $\mathrm{Co}_{90} \mathrm{Fe}_{10} / \mathrm{Pd}$ multilayers by use of broadband ferromagnetic resonance spectroscopy, Phys. Rev. B 85, 054412 (2012).

[45] M. Oogane et al., Magnetic damping in ferromagnetic thin films, Jpn. J. Appl. Phys. 45, 3889 (2006).

[46] C. Scheck, L. Cheng, and W. E. Bailey, Low damping in epitaxial sputtered iron films, Appl. Phys. Lett. 88, 252510 (2006).

[47] Y. Yin et al., Tunable permalloy-based films for magnonic devices, Phys. Rev. B 92, 024427 (2015).

[48] J. M. Shaw, T. J. Silva, M. L. Schneider, and R. D. McMichael, Spin dynamics and mode structure in nanomagnet arrays: Effects of size and thickness on linewidth and damping, Phys. Rev. B 79, 184404 (2009). 
[49] S. M. Bhagat and P. Lubitz, Temperature variation of ferromagnetic relaxation in the $3 d$ transition metals, Phys. Rev. B 10, 179 (1974).

[50] J. Pelzl et al., Spin-orbit-coupling effects on $g$ value and damping factor of the ferromagnetic resonance in $\mathrm{Co}$ and $\mathrm{Fe}$ films, J. Phys.: Condens. Matter 15, S451 (2003).

[51] C. T. Boone, H. T. Nembach, J. M. Shaw, and T. J. Silva, Spin transport parameters in metallic multilayers determined by ferromagnetic resonance measurements of spin-pumping, J. Appl. Phys. 113, 153906 (2013).

[52] C. T. Boone, J. M. Shaw, H. T. Nembach, and T. J. Silva, Spinscattering rates in metallic thin films measured by ferromagnetic resonance damping enhanced by spin-pumping, J. Appl. Phys. 117, 223910 (2015)

[53] J. M. Shaw, H. T. Nembach, and T. J. Silva, Damping phenomena in $\mathrm{Co}_{90} \mathrm{Fe}_{10} /$ Nimultilayers and alloys, Appl. Phys. Lett. 99, 012503 (2011).

[54] F. D. Czeschka et al., Scaling Behavior of the Spin Pumping Effect in Ferromagnet-Platinum Bilayers, Phys. Rev. Lett. 107, 046601 (2011).

[55] M. Weiler, J. M. Shaw, H. T. Nembach, and T. J. Silva, Detection of the dc inverse spin Hall effect due to spin pumping in a novel meander-stripline geometry, Magn. Lett., IEEE 5, 1 (2014).

[56] J. Walowski et al., Intrinsic and non-local Gilbert damping in polycrystalline nickel studied by Ti:sapphire laser fs spectroscopy, J. Phys. D 41, 164016 (2008).

[57] T. Qu and R. H. Victora, Dependence of Kambersky damping on Fermi level and spin orientation, J. Appl. Phys. 115, 17C506 (2014).
[58] S. Ingvarsson, L. Ritchie, X. Y. Liu, G. Xiao, J. C. Slonczewski, P. L. Trouilloud, and R. H. Koch, Role of electron scattering in the magnetization relaxation of thin $\mathrm{Ni}_{81} \mathrm{Fe}_{19}$ films, Phys. Rev. B 66, 214416 (2002).

[59] C. Luo et al., Enhancement of magnetization damping coefficient of permalloy thin films with dilute Nd dopants, Phys. Rev. B 89, 184412 (2014).

[60] K. Kobayashi et al., Damping constants for permalloy singlecrystal thin films, IEEE Trans. Magn. 45, 2541 (2009).

[61] Y. Zhao et al., Experimental investigation of temperaturedependent Gilbert damping in permalloy thin films, Sci. Rep. 6, 22890 (2016).

[62] Y. Liu, Z. Yuan, R. J. H. Wesselink, A. A. Starikov, and P. J. Kelly, Interface Enhancement of Gilbert Damping from First Principles, Phys. Rev. Lett. 113, 207202 (2014).

[63] H. Ebert, S. Mankovsky, D. Ködderitzsch, and P. J. Kelly, Ab Initio Calculation of the Gilbert Damping Parameter via the Linear Response Formalism, Phys. Rev. Lett. 107, 066603 (2011).

[64] S. Lounis, M. dos Santos Dias, and B. Schweflinghaus, Transverse dynamical magnetic susceptibilities from regular static density functional theory: Evaluation of damping and $g$ shifts of spin excitations, Phys. Rev. B 91, 104420 (2015).

[65] T. Devolder et al., Damping of $\mathrm{Co}_{x} \mathrm{Fe}_{80-x} \mathrm{~B}_{20}$ ultrathin films with perpendicular magnetic anisotropy, Appl. Phys. Lett. 102, 022407 (2013).

[66] V. Kamberský, On the Landau-Lifshitz relaxation in ferromagnetic metals, Can. J. Phys. 48, 2906 (1970).

[67] R. J. Elliott, Theory of the effect of spin-orbit coupling on magnetic resonance in some semiconductors, Phys. Rev. 96, 266 (1954). 